\title{
A Note from the Editor and the Publisher
}

We are delighted to announce that Anthropology in Action (AiA) will be published as an open access journal as of 2018. Thanks to the generous support that we have received from a global network of libraries as part of the Knowledge Unlatched Select programme, there are no submission charges or article-processing charges (APCs) for authors of articles published under this arrangement. ${ }^{1}$ The initial funding is for three years (2018-2020), and during this time we will also make the backfiles of the journal freely available. This is an exciting moment not just for the journal but for its authors, as it offers them a great opportunity to further enhance the reach of their articles. We greatly appreciate the support of Knowledge Unlatched and its Title Selection Committee in choosing AiA and would like to express our thanks to the supporting institutions whose collective resources have removed the financial burden of open access from the journal's authors.

There are many benefits to open access, especially for a journal like AiA, whose applied approach to anthropology finds resonance in many fields outside of anthropology, where access to anthropology resources may be limited. Yet, despite the proliferation of open access mandates for research, sustainable open access models remain a challenge and ultimately a barrier for many scholars. This is especially the case for many of our authors, for whom access to APC funds are limited, as is often the case for research funding in general across the social sciences and the humanities. Therefore, we are encouraged by the collaborative efforts driven by Knowledge Unlatched, which brings together libraries to pool their resources to fund the books and journals they feel merit their support. To be selected by a community of respected scholarly librarians is a testament to the mission of AiA, and we are very pleased about the benefit that this programme will bring to our authors.

We anticipate that the open access benefit of this programme will be very attractive to many authors, and the Editors encourage you to submit your contributions accordingly for publication consideration. However, while the move to open access changes the form of distribution of the articles published starting this year, there is no change to the submission process, which will continue to be handled through our traditional route as outlined on the 'Info for Authors' tab on the AiA website, where you can find guidelines and instructions. ${ }^{2}$ Articles must be just as relevant to our remit and scope as before, and will continue to undergo the same thorough peer-review process that has long been established for our selection process. No preferential treatment or scheduling will be given to anyone for whom open access is a condition.

We look forward to continuing to bring our readers articles that foster the broader application of anthropological approaches to practical problems, now with further opportunities for the interdisciplinary exchange of research within and outside of academia that $\mathrm{AiA}$ advocates thanks to open access.

Christine McCourt

April 2018

\section{Notes}

1. http://www.knowledgeunlatched.org/ku-selectlibrary-info/.

2. www.berghahnjournals.com/aia. 
